\title{
Sondas endopleurales en trauma torácico no quirúrgico. Experiencia en un centro de trauma de nivel I
}

\author{
Alberto Pérez Cantú-Sacal, $\bowtie$ Demian Trueba-Lozano, Jonathan García-Esqueda, \\ Juan Carlos Vázquez-Minero
}

Hospital Central de la Cruz Roja Mexicana, Ciudad de México.

Trabajo recibido: 07-VII-2014; aceptado: 11-XI-2014

\begin{abstract}
RESUMEN. Introducción: Un tercio de las lesiones torácicas amerita admisión hospitalaria, y la inserción de una sonda endopleural es el único procedimiento invasivo que requiere la mayoría ( > 85\%). Entre 7.6-30\% de los pacientes sometidos a este procedimiento, experimenta alguna complicación. Material y métodos: Se trata de un estudio retrospectivo, descriptivo y observacional realizado en el Hospital Central de la Cruz Roja Mexicana, donde se revisaron los expedientes de todos los pacientes que ingresaron durante el período comprendido de febrero de 2007 a diciembre de 2013, con antecedente de traumatismo contuso o penetrante y cuyas lesiones ameritaron inserción de sonda endopleural. Resultados: Se incluyeron 812 pacientes, de éstos, 758 hombres y 54 mujeres de 18-45 años de edad; 68 tenían antecedente de traumatismo torácico contuso y 744 de traumatismo penetrante. La frecuencia de las lesiones que fueron consideradas como indicación para insertar sonda endopleural fue la siguiente: 206 neumotórax, 249 hemotórax y 357 hemoneumotórax. El tiempo promedio de permanencia de las sondas fue de 4.53 días y se registraron 13 (1.60\%) complicaciones, 8 neumotórax residuales que ameritaron reinserción de la sonda y 5 hemotórax retenidos que ameritaron efectuar toracotomía para su resolución. Discusión: Los traumatismos torácicos predominaron en la población masculina con una relación de 14:1, la mayoría (91.62\%) con mecanismo penetrante. La ocurrencia de complicaciones en relación con la cavidad pleural fue evaluada en el período comprendido entre la colocación de la sonda endopleural y la primer consulta una semana después de su retiro; éstas se observaron únicamente en $1.60 \%$ de los pacientes y sólo $0.61 \%$ requirieron cirugía, implicando una reducción de 78.95 y $93.90 \%$ con respecto de los estudios analizados. Además, el tiempo promedio de permanencia de las sondas fue de 4.53 días, es decir, $30.95 \%$ menos que en la literatura consultada. Conclusiones: Nuestro protocolo para el manejo de sondas endopleurales en situaciones de trauma ha demostrado ser una técnica efectiva con complicaciones inherentes prácticamente nulas.
\end{abstract}

Palabras clave: Sonda endopleural, trauma torácico, hemotórax, neumotórax.

ABSTRACT. Introduction: One third of thoracic injuries require hospitalization and the insertion of a chest drain is the only invasive procedure needed most of the times ( $>85 \%$ ). Between $7.6-30 \%$ of patients undergoing this procedure experience some complication. Material and methods: A retrospective, descriptive, observational study was conducted at the Central Hospital of the Mexican Red Cross with the review of the records of all patients admitted during the period of February 2007 to December 2013 with history of blunt or penetrating trauma and whose injuries required the insertion of a chest drain. Results: 812 patients were included -758 men and 54 women-, between $18-45$ years old; 68 had history of blunt chest trauma and 744 of penetrating trauma. The frequency of the injuries that were considered as an indication to insert a chest drain was: 206 pneumothorax, 249 hemothorax and 357 hemopneumothorax. The average dwelling time of the drains was 4.53 days and there were $13(1.60 \%)$ complications recorded, 8 residual pneumothorax needing drain reinsertion and 5 retained hemothorax requiring thoracotomy for resolution. Discussion: Thoracic injuries predominated among males with a ratio of $14: 1$, most (91.62\%) with penetrating mechanism. The occurrence of complications related to the pleural cavity was evaluated in the period between the placement of the chest drain and the first consultation a week after its withdrawal; these were observed only in $1.60 \%$ of patients and only $0.61 \%$ of them required surgery, involving a reduction of 78.95 and $93.90 \%$ in comparison with the studies analyzed; in addition, the average dwelling time of the probes was 4.53 days, i.e. $30.95 \%$ less than in the reviewed literature. Conclusions: Our protocol for handling chest drains in trauma situations has proven to be an effective technique with almost no inherent complications.

Key words: Chest drain, chest trauma, pneumothorax, hemothorax.

\section{INTRODUCCIÓN}

El tórax constituye casi la cuarta parte de la masa corporal; este hecho implica que con frecuencia sea sujeto de lesiones por traumas que ameriten una evaluación lógica y secuencial, seguida de la terapéutica específica, la cual puede implicar una cirugía ( $<20 \%$ del total, $<10 \%$ de los traumatismos contusos y $15-30 \%$ de los traumatismos penetrantes) $\cdot^{1-3}$ Las lesiones que amenazan la vida de manera inmediata, como consecuencia 
del suministro insuficiente de sangre oxigenada al cerebro y otras estructuras vitales, deben de ser tratadas lo más sencilla y rápidamente posible. ${ }^{4,5}$

En condiciones normales, la cavidad pleural mantiene una presión negativa con respecto de la atmosférica, la cual facilita la expansión pulmonar durante la inspiración profunda; sin embargo, cuando existe una colección, se restringe la expansión pulmonar. Por esta razón, los traumatismos torácicos son una causa importante de mortalidad ( $>25 \%$ de las muertes relacionadas con trauma). ${ }^{1,4-7}$

Alrededor de un tercio de las lesiones torácicas amerita admisión hospitalaria, y la inserción de una sonda endopleural es el único procedimiento invasivo que requiere la mayoría ( $>85 \%$ ). ${ }^{1,3}$ Hipócrates fue el primero en describir la descompresión torácica en casos de empiema; y cuando en 1891 Gotthard Bülau introdujo el drenaje pleural continuo mediante succión a través de una sonda, se convirtió en el procedimiento de rutina para el drenaje de empiema, especialmente en pacientes con tuberculosis. A partir de la guerra de Corea, las sondas endopleurales empezaron a ser utilizadas para el tratamiento de emergencia de lesiones torácicas. ${ }^{8}$

Entre $7.6-30 \%$ de los pacientes sometidos a colocación de sonda endopleural enfrentan alguna dificultad relacionada con la inserción (7.9\%), la posición inadecuada (5.2-16\%), ${ }^{3,5}$ la conexión al sistema de drenaje torácico, la presión en el dispositivo de sello de agua o al momento de retirarla, las cuales pueden resultar en hemotórax coagulado, neumotórax residual o empiema; ante ello, la estancia hospitalaria de muchos pacientes únicamente depende del tiempo que permanecen con la sonda endopleural insertada. Por otra parte, con demasiada frecuencia se emplean sondas por segunda ocasión (de manera innecesaria) como consecuencia del conocimiento erróneo sobre su función y la técnica para insertarlas. Los factores de riesgo que han sido identificados para el desarrollo de complicaciones como consecuencia de la inserción de una sonda endopleural incluyen: procedimiento efectuado por personal ajeno al área quirúrgica, procedimiento efectuado fuera del área de trauma y choque, paciente con necesidad de apoyo ventilatorio mecánico, paciente con estado de choque al ingreso en el Departamento de Urgencias y paciente con requerimiento de manejo en Unidad de Cuidados Intensivos. , $^{2,4,6,9-11}$

Cuando se evalúa y proporciona tratamiento a pacientes con trauma torácico es necesario reconocer que a pesar de su larga historia de uso clínico, el rol y el manejo de las sondas endopleurales y sus sistemas de drenaje permanecen definidos de manera incompleta, pues la variedad de materiales con los cuales se fabrican las sondas, las diferencias en su diseño y el de las unidades de drenaje y la cantidad de indicaciones para su empleo contribuyen a que surjan problemas, como la elección de un tamaño de sonda inapropiado para la colección que se desea drenar, la costumbre de pinzar las sondas con el consecuente riesgo de desarrollar neumotórax a tensión o edema pulmonar por reexpansión, efectuar su retiro sin la analgesia apropiada y retrasar su retiro en pacientes con apoyo ventilatorio mecánico. ${ }^{2,6,7,11-13}$

A continuación, presentamos un revisión de la experiencia en el manejo de sondas endopleurales en trauma torácico no quirúrgico en un centro de trauma de nivel I.

\section{MATERIAL Y MÉTODOS}

Se trata de un estudio retrospectivo, descriptivo y observacional que se llevó a cabo en el Hospital Central de la Cruz Roja Mexicana de la Ciudad de México, fueron revisados todos los expedientes de los pacientes de ambos sexos, masculino y femenino, y con un rango de edad de 18 a 45 años que ingresaron durante el período comprendido de febrero de 2007 a diciembre de 2013, con antecedente de traumatismo torácico contuso o penetrante y cuyas lesiones diagnosticadas de manera clínica o mediante estudios de gabinete: radiografía de tórax (figura 1), tomografía de tórax o eFAST (del inglés extended Focused Abdominal Sonography for Trauma o ultrasonido en trauma ampliado), ${ }^{14}$ ameritaron inserción de sonda endopleural para su resolución. Fueron excluidos los pacientes con necesidad de toracotomía de urgencia por hemotórax masivo, que no tuvieron indicación de colocación de sonda endopleural, que fueron trasladados a otro hospital antes de efectuar el

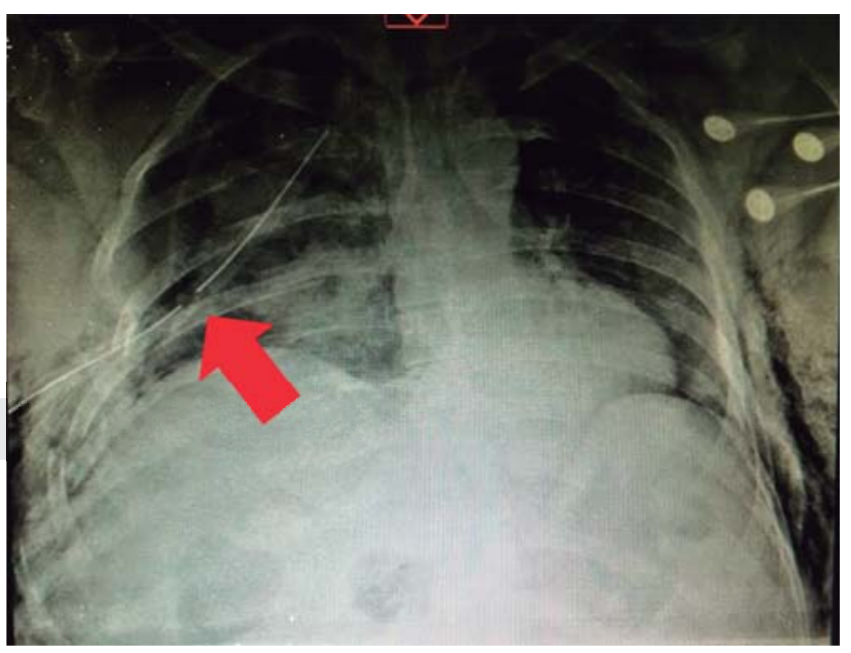

Figura 1. Radiografía de tórax de control poscolocación de sonda endopleural (flecha). 


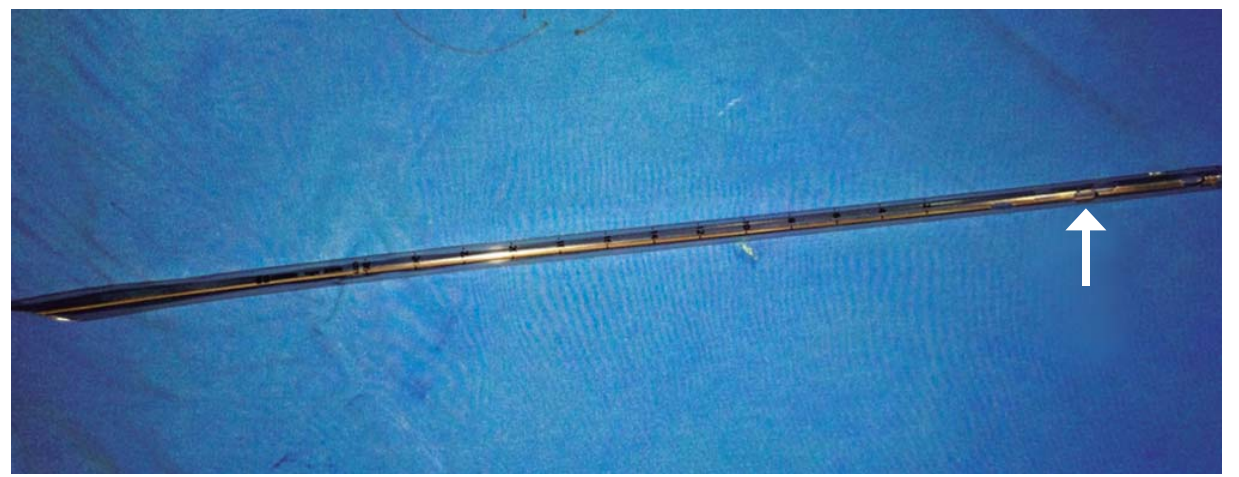

Figura 2.

Sonda endopleural $32 \mathrm{Fr}$ fenestrada (flecha) radioopaca. retiro de la sonda y contar con evidencia radiográfica de ausencia de complicaciones o que ameritaron inserción de sonda endopleural como consecuencia de una toracotomía programada.

Las variables tomadas en cuenta para este estudio fueron sexo y edad del paciente, tipo y mecanismo de lesión, tiempo de permanencia de la sonda, días de estancia hospitalaria, controles radiográficos para evaluar el retiro exitoso de la sonda y complicaciones inherentes al procedimiento, tales como hemotórax coagulado, neumotórax residual o empiema. Los datos fueron extraídos del expediente clínico. Se realizó análisis de variables cuantitativas.

En todos los pacientes se emplearon sondas Argyle $^{\mathrm{TM}}$ Thoracic Catheter Straight $32 \mathrm{Fr}(10.7 \mathrm{~mm})$ x 20" $(51 \mathrm{~cm})$ (figura 2), las cuales fueron colocadas entre las líneas axilar anterior y media en el quinto espacio intercostal (figuras 3 y 4), fijadas con seda 1 a la piel (figura 5) y conectadas a un sistema de drenaje torácico de tres cámaras Atrium ${ }^{\text {TM }}$ Ocean Water Seal Chest 2002 Single Collection (figura 6) con succión continua a $20 \mathrm{cmH}_{2} \mathrm{O}$. Durante el procedimiento se les administró tramadol 100 mg por vía intravenosa e infiltró en el sitio de colocación con lidocaína al $2 \%$ con epinefrina a una dosis de 7 $\mathrm{mg} / \mathrm{kg}$, después se les proporcionó antibioticoterapia con ceftriaxona $1 \mathrm{~g}$ cada 12 horas por vía intravenosa durante el período de permanencia de la sonda y analgesia con ibuprofeno $400 \mathrm{mg}$ cada ocho horas por vía enteral si estaba disponible la misma, hasta el retiro de la sonda; además, recibieron curación diaria del sitio de inserción de la sonda - consistente en lavado del sitio quirúrgico con iodopovidona y movilización gentil de la sonda, verificando su permeabilidad-bajo analgesia con tramadol y con posterior control radiográfico.

Los criterios para retirar la sonda endopleural fueron: ausencia de dificultad respiratoria, gasto de material seroso (sin trazos hemáticos) en cantidad menor de $150 \mathrm{cc}$, evidencia radiológica de completa expansión pulmonar sin colecciones pleurales; y en el caso de

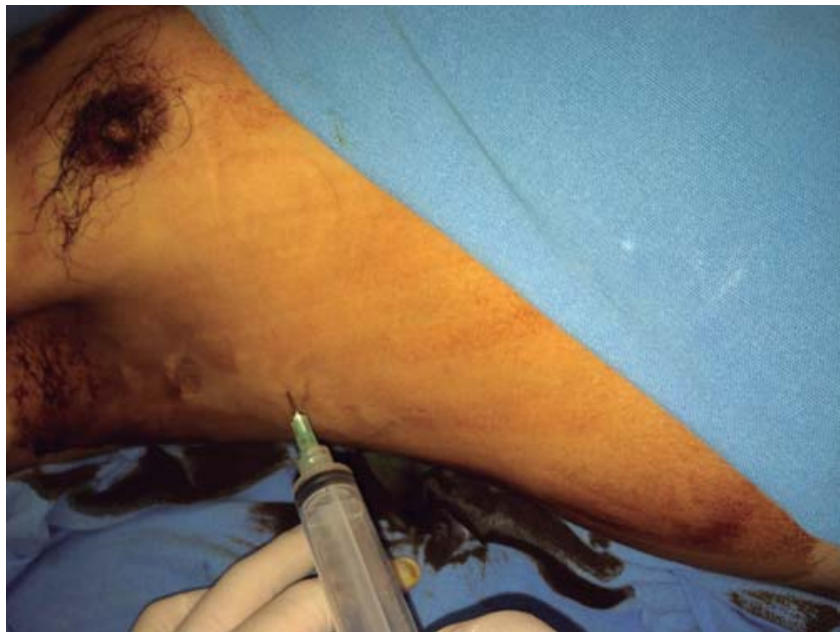

Figura 3. Sitio de colocación de la sonda endopleural: infiltración con anestésico.

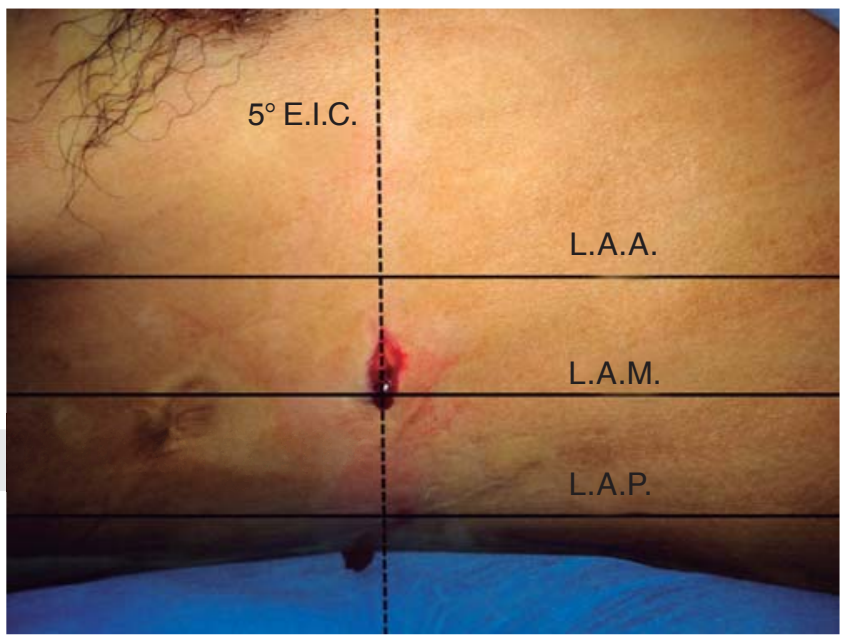

Figura 4. Sitio de colocación de la sonda endopleural: incisión en el quinto espacio intercostal ( $\left.5^{\circ} \mathrm{EIC}\right)$, línea axilar media (LAM). Línea axilar anterior (LAA), línea axilar posterior (LAP). 


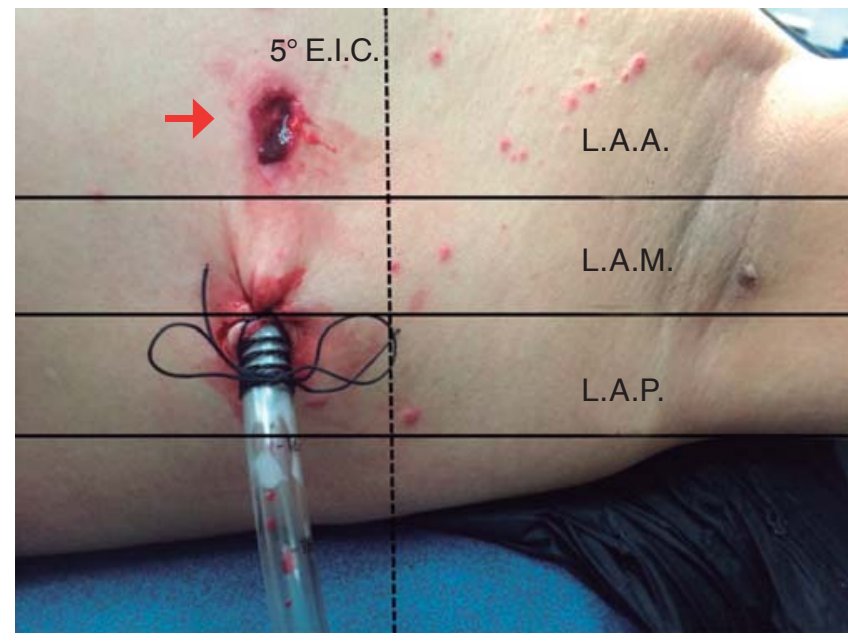

Figura 5. Sitio de colocación de la sonda endopleural: fijación de la sonda. Flecha (orificio de entrada de proyectil de arma de fuego), $5^{\circ}$ EIC (5० espacio intercostal), LAA (línea axilar anterior), LAM (línea axilar media), LAP (línea axilar posterior).

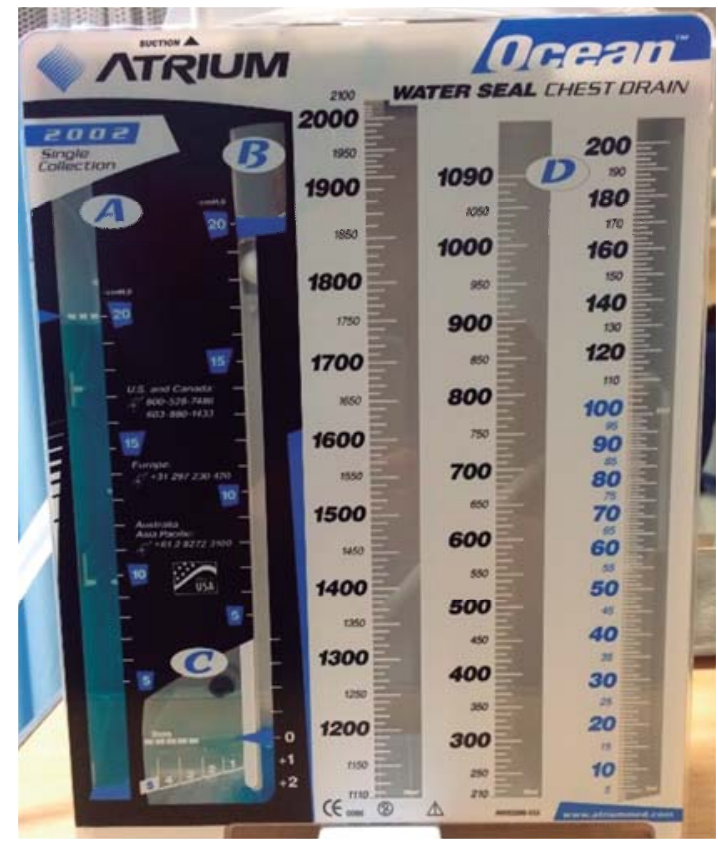

Figura 6. Sistema de drenaje torácico.

pacientes con apoyo ventilatorio mecánico, presión al final de la espiración (PEEP) $\leq 4 \mathrm{cmH}_{2} \mathrm{O}$ y parámetros gasométricos compatibles con un índice de Kirby $>300$ en ausencia de acidosis o alcalosis respiratoria. El retiro de la sonda se efectuó bajo analgesia con tramadol en espiración forzada y manteniendo la succión continua hacia el sistema de drenaje torácico, solicitando estudio radiográfico de control seis horas después para corroborar el éxito del procedimiento.
Tabla 1. Mecanismo de lesión de los pacientes que ameritaron inserción de sonda pleural en el período 2007-2013.

\begin{tabular}{ccc}
\hline Mecanismo & Cantidad & Porcentaje \\
\hline Contuso & 68 & 8.37 \\
AVM $^{*}$ & 59 & 86.76 \\
Caídas $^{*}$ & 7 & 10.29 \\
Aplastamiento $^{\text {Penetrante }}$ & 2 & 2.94 \\
HPIC $^{\ddagger}$ & 744 & 91.62 \\
HPAF $^{\S}$ & 472 & 58.12 \\
\hline
\end{tabular}

*Accidente en vehículo automotor. cortante. ${ }^{\S}$ Herida por proyectil de arma de fuego.

\section{RESULTADOS}

Se incluyeron 812 pacientes 758 (94.27\%) hombres y 54 $(6.65 \%)$ mujeres de 18 a 45 años de edad, con media de 29.81 años, 68 (8.37\%) tenían antecedente de traumatismo torácico contuso y $744(91.62 \%)$ de traumatismo penetrante, $472(58.12 \%)$ por herida por instrumento punzocortante y $272(33.49 \%)$ por herida por proyectil de arma de fuego. 459 (56.52\%) tenía otras lesiones asociadas: 298 en extremidades (36.69\%), 106 en abdomen $(23.09 \%)$ y 55 en cráneo (11.98\%) (Tabla 1).

Las indicaciones para insertar una sonda endopleural fueron: 206 (25.36\%) por neumotórax, 27 (3.52\%) por trauma contuso y 179 (22.04\%) por trauma penetrante; 249 (30.66\%) hemotórax, 25 (3.07\%) por trauma contuso y 224 (27.58\%) por trauma penetrante; y 357 $(43.96 \%)$ hemoneumotórax, 16 (1.97\%) por trauma contuso y 341 (41.99\%) por trauma penetrante.

El tiempo promedio de permanencia de las sondas endopleurales fue de 4.53 días, con un mínimo de dos días y máximo de 20 días; el tiempo de estancia hospitalaria osciló entre 2 y 60 días, con un promedio de 4.98 días. La ocurrencia de complicaciones en relación con la cavidad pleural fue evaluada en el período comprendido entre la colocación de la sonda endopleural y la primera consulta, una semana después de su retiro; se registraron $13(1.60 \%)$ complicaciones: 8 (61.53\%) neumotórax residuales que ameritaron reinserción de la sonda y 5 (38.46\%) hemotórax retenidos que ameritaron efectuar toracotomía para su resolución. Se evaluaron sólo las complicaciones tempranas y las evaluadas en la consulta externa donde se atendió a todos los pacientes.

\section{DISCUSIÓN}

En nuestro estudio encontramos predominio de traumatismos torácicos en la población masculina 
con una relación de 14:1, la gran mayoría con mecanismo penetrante $(91.62 \%)$ como consecuencia de herida por instrumento punzocortante (58.12\%) y con hemotórax o hemoneumotórax concomitante (74.63\%). En la literatura consultada se afirma que más de la mitad de los traumatismos afecta a la población masculina y casi la cuarta parte compromete el tórax, que después de las fracturas costales el neumotórax constituye la manifestación más común de lesión torácica (40-50\%) y que cerca de $20 \%$ de los pacientes con trauma torácico o múltiple tiene hemotórax concomitante. 1,7,8,12 Las diferencias encontradas no resultan sorprendentes si consideramos que nuestro hospital recibe principalmente pacientes traumatizados, muchos de ellos como consecuencia de agresión por terceras personas.

En nuestro hospital, las complicaciones en relación con la cavidad pleural ocurrieron únicamente en $1.60 \%$ de los pacientes y sólo $0.61 \%$ de ellos requirieron cirugía, implicando una reducción de 78.95 y $93.90 \%$ con respecto de los estudios analizados. Además, nuestro tiempo promedio de permanencia de las sondas fue de 4.53 días; es decir, $30.95 \%$ menos que en la literatura consultada, donde se afirma que $7.6-30 \%^{3,5}$ de los pacientes sometidos a la colocación de una sonda endopleural sufrirá alguna complicación —con necesidad de resolución quirúrgica en $<10 \%$ de los traumatismos cerrados y $15-30 \%$ de los traumatismos penetrantes (< $20 \%$ del total) $)^{1,2,4}$ - y se reporta un tiempo promedio de permanencia de 6.56 días. ${ }^{1}$

Para la elección del tamaño apropiado de una sonda endopleural se considera que su diámetro, y en menor medida su longitud, son los determinantes más importantes de la velocidad de flujo en su interior; sin embargo, es necesario tomar en cuenta qué clase de material es el que se desea drenar y la velocidad con la cual se genera. En este sentido y para fines prácticos, puede afirmarse que la producción continua y rápida de líquidos viscosos, como la sangre, amerita el empleo de sondas de mayor diámetro que las que se utilizarían para drenar un volumen similar de aire.7,8,12 Tradicionalmente, en el Hospital Central de la Cruz Roja Mexicana se han utilizado sondas de calibre $32 \mathrm{Fr}$, pues es un tamaño cómodo para evacuar neumotórax, hemotórax y hemoneumotórax traumáticos.

Respecto al manejo del sistema de drenaje torácico, en la literatura consultada se menciona que el aire y el líquido libre en la cavidad pleural suelen drenar del tórax sin necesidad de succión debido a la fuerza de gravedad - por lo cual sólo deberá emplearse succión $\left(<20 \mathrm{cmH}_{2} \mathrm{O}\right)$ en caso de que por algún motivo no respondan de manera adecuada-, que sólo existen dos indicaciones para cambiar o vaciar el dispositivo de sello de agua; es decir, que la cámara para colectar contenga fluido turbio o se encuentre llena, y que en caso de requerir mover al paciente, el dispositivo siempre debe mantenerse por debajo del nivel del tórax con el fin de prevenir el retorno del fluido. ${ }^{7,12}$ En nuestra institución siempre empleamos sistemas de drenaje torácico de tres cámaras con succión continua a $20 \mathrm{cmH}_{2} \mathrm{O}$.

El volumen del gasto de una sonda endopleural considerado seguro para retirarla parece haber sido establecido de manera arbitraria y no con base en la evidencia; en este sentido, el estudio de Younes et al. - citado por Baumann- no encontró diferencias significativas en tiempo de permanencia de la sonda, estancia hospitalaria, acumulación de fluido y necesidad de toracentesis en pacientes a quienes les fueron retiradas las sondas endopleurales con gastos de $\leq 100$ $\mathrm{mL} /$ día, $\leq 150 \mathrm{~mL} /$ día o $200 \mathrm{~mL} /$ día. $^{5,12,15}$ Los criterios de seguridad que tomamos en cuenta para efectuar el retiro de las sondas son gasto de material seroso en cantidad menor de $150 \mathrm{~cm}^{3}$ (pues la producción de líquido pleural ocurre a una velocidad de $0.1-0.2 \mathrm{~cm}^{3} /$ $\mathrm{kg} / \mathrm{h}$, que en un individuo promedio de $70 \mathrm{~kg}$ equivale a $\left.168-336 \mathrm{~cm}^{3}\right) ; 16$ esto aunado a los demás criterios antes mencionados pareció ofrecer un margen protocolizado seguro para el retiro de todas las sondas endopleurales obteniendo una tasa baja de necesidad de recolocación o complicaciones a consecuencia de retiro de la misma por razones erróneas o mal justificadas clínicamente.

La literatura disponible menciona dos métodos seguros para efectuar el retiro de sondas endopleurales: el primero consiste en retirar la sonda empleando succión; el segundo implica descontinuar la succión mientras la sonda permanece conectada al dispositivo de sello de agua durante 18-24 horas y solicitar un estudio radiológico de control antes de efectuar el retiro. Quienes favorecen el primer método, mencionan que ofrece la ventaja de proporcionar succión durante todo el proceso de retiro, disminuyendo la recurrencia de neumotórax; los que prefieren el segundo, afirman que facilita la detección de fuga aérea oculta, procurando - aparentemente- el mismo beneficio. A pesar de que no se evidencian diferencias significativas entre ambos métodos en cuanto al desarrollo de neumotórax residual, el estudio de Martino et al. señala que en caso de existir la necesidad de insertar otra sonda endopleural, es mayor en el grupo de pacientes sometidos a retiro con el primer método (neumotórax residual $\geq 20 \%$ ). ${ }^{5,10-12}$

También existen opiniones que favorecen la práctica de retirar las sondas al final de la inspiración o al final de la espiración. Los que favorecen el primer método argumentan que al final de la inspiración, el pulmón se encuentra completamente expandido y, en consecuencia, las pleuras parietal y visceral mantienen un 
contacto más íntimo, impidiendo la entrada de aire. Los que se inclinan por el segundo método aducen que al final de la espiración, la diferencia entre las presiones de la atmósfera y del espacio pleural es menor y, por lo tanto, disminuye el riesgo de que penetre aire en la cavidad torácica. Al respecto, la valoración efectuada por Bell et al. (citada igualmente por Baumann) no encontró diferencias significativas entre ambos métodos en cuanto a la recurrencia de neumotórax ni demostró factores que predispusieran a esta complicación.10,11,13-15 En nuestro hospital, el retiro de las sondas se efectúa bajo analgesia con tramadol, en espiración forzada y manteniendo la succión continua hacia el sistema de drenaje torácico, solicitando telerradiografía de tórax de control seis horas después para corroborar el éxito del procedimiento. ${ }^{12,16-19}$

La incidencia de empiema postraumático en pacientes que sufrieron lesiones en el tórax oscila entre 2-25\%; el microorganismo causal que se aísla con mayor frecuencia es Staphylococcus aureus. Se han identificado como causas potenciales la infección iatrogénica del espacio pleural durante la inserción de la sonda endopleural, infección directa como consecuencia de lesiones penetrantes en la cavidad torácica, infección secundaria asociada con lesión de órganos intraabdominales con disrupción diafragmática, permanencia de la sonda mayor de seis días, estancia en unidad de cuidados intensivos mayor de dos días, infección secundaria de hemotórax drenado de manera inadecuada, diseminación hematógena o linfática de infecciones subdiafragmáticas y empiema paraneumónico resultante de neumonía postraumática, contusión pulmonar o síndrome de distrés respiratorio agudo. Otra controversia que enfrenta el personal a cargo del paciente traumatizado ocurre con el empleo de antibióticos «profilácticos» en los pacientes con sonda endopleural (el uso de comillas se justifica pues, considerando que en la mayor parte de los casos la sonda se inserta antes de iniciar la administración de antibióticos, sería más apropiado el término «tratamiento antibiótico presuntivo»). El estudio efectuado por Eren et al., sólo recomienda el empleo de antibióticos en caso de identificar alguno de los factores de riesgo para el desarrollo de empiema postraumático mencionados supra, mientras que la guía de práctica basada en evidencia publicada por Luchette et al. y el editorial acompañante de Wilson y Nichols referentes a sondas endopleural colocadas en situaciones de trauma, ofrecen recomendaciones sustentadas en todos los niveles de evidencia a favor del empleo de una cefalosporina de primera generación durante un período menor de 24 horas, sugiriendo que esta práctica podría reducir la incidencia de neumonía, aunque no la de empiema. Por otra parte, el estudio de Karmy-Jones et al. sugiere que basta con efectuar la inserción de la sonda mediante técnica estéril y evacuar completamente el hemotórax con el fin de prevenir el desarrollo de empiema. ${ }^{20-24}$ En nuestro hospital, siempre se emplea antibioticoterapia con ceftriaxona $1 \mathrm{~g}$ cada 12 horas por vía intravenosa durante siete días y la incidencia de empiema postraumático es de $0 \%$.

\section{CONCLUSIONES}

La inserción urgente de una sonda endopleural reduce las tasas de morbimortalidad como consecuencia de traumatismos torácicos, el diagnóstico temprano de las lesiones que se beneficiarán de este procedimiento es de suma importancia para evitar complicaciones. El protocolo que hemos adoptado para llevar a cabo la colocación de sondas endopleurales en nuestro hospital ha demostrado ser efectiva para lograr complicaciones inherentes al evento quirúrgico prácticamente nulas.

Si bien el diseño del estudio en el cual se excluyeron pacientes > 45 años (y por lo tanto con mayor probabilidad de morbilidad previa al trauma) y aquéllos que requirieron cirugía inmediata (es decir, con lesiones más graves) implica un sesgo que puede explicar la morbilidad sorprendentemente baja que encontramos, también existe una relación con nuestra práctica habitual de efectuar curación diaria de las sondas endopleurales, la cual permite evitar hemotórax retenidos y coagulados y empiemas, así como intervenciones quirúrgicas como consecuencia de la oclusión de las sondas por coágulos. Este protocolo no es una práctica habitual en otras instituciones y no es mencionado en la literatura consultada como parte de los cuidados que deben ser proporcionados al paciente después de ser sometido a la inserción de una sonda endopleural. Consideramos que se trata de una variable particular de nuestro estudio.

Por último, debemos puntualizar que a diferencia de lo reportado en otras series, nuestra experiencia es vasta y describimos una técnica fácilmente reproducible, por lo que recomendamos tomar en consideración nuestro protocolo para un adecuado proceso diagnóstico y terapéutico en traumatismos de tórax, de tal manera que el manejo del paciente, su alta hospitalaria y su recuperación se encuentren libres de complicaciones.

\section{REFERENCIAS}

1. Villegas-Carlos F, Vázquez-Martínez AM, PinedoOnofre JA, Guevara-Torres L, Belmares-Taboada JA, Sánchez-Aguilar M. Utilidad de los antimicrobianos 
en la toracostomía cerrada por trauma. Cir Ciruj 2009;77(1):29-32.

2. Mattox KL, Matthew JW, Tsai P. Trauma thoracotomy: principles and techniques. In: Mattox $\mathrm{KL}$, Moore $\mathrm{EE}$, Feliciano DV, editors. Trauma. 7th ed. United States: McGraw-Hill; 2013. p. 461-467.

3. Fitzgerald M, Mackenzie CF, Marasco S, Hoyle R, Kossmann T. Pleural decompression and drainage during trauma reception and resuscitation. Injury 2008;39(1):920. doi: 10.1016/j.injury.2007.07.021.

4. Colegio Americano de Cirujanos. Comité de trauma. Programa avanzado de apoyo vital en trauma. Manual del curso para estudiantes. 9a. ed. Estados Unidos: American College of Surgeons; 2012.

5. Menger R, Telford G, Kim P, et al. Complications following thoracic trauma managed with tube thoracostomy. Injury 2012;43(1):46-50. doi: 10.1016/j. injury.2011.06.420.

6. Rivera L, O'Reilly EB, Sise MJ, et al. Small catheter tube thoracostomy: effective in managing chest trauma in stable patients. J Trauma 2009;66(2):393-399. doi: 10.1097/TA.0b013e318173f81e.

7. Durai R, Hoque H, Davies TW. Managing a chest tube and drainage system. AORN J 2010;91(2):275-280; quiz 281-3. doi: 10.1016/j.aorn.2009.09.026.

8. Huber-Wagner S, Körner M, Ehrt A, et al. Emergency chest tube placement in trauma care-which approach is preferable? Resuscitation 2007;72:226-233.

9. Maritz D, Wallis L, Hardcastle T. Complications of tube thoracostomy for chest trauma. S Afr Med J 2009;99(2):114-117.

10. Martino K, Merrit S, Boyakye K, et al. Prospective randomized trial of thoracostomy removal algorithms. J Trauma 1999;46(3):369-371.

11. Tawil I, Gonda JM, King RD, Marinaro JL, Crandall CS. Impact of positive pressure ventilation on thoracostomy tube removal. J Trauma 2010;68(4):818-821. doi: 10.1097/TA.0b013e3181a5973c.

12. Baumann $\mathrm{MH}$. What size chest tube? What drainage system is ideal? And other chest tube management questions. Curr Opin Pulm Med 2003;9(4):276-281.

13. Tsai WK, Chen W, Lee JC, et al. Pigtail catheter vs largebore chest tubes for management of secondary spontaneous pneumothoraces in adults. Am J Emerg Med 2006;24(7):795-800.

14. Flato UA, Guimarães HP, Lopes RD, Valiatti JL, Flato EM, Lorenzo RG. Usefulness of extended-FAST (EFAST-
Extended Focused Assessment with Sonography for Trauma) in critical care setting. Rev Bras Ter Intensiva 2010;22(3):291-299.

15. Bell RL, Ovadia P, Abdullah F, Spector S, Rabinovici R. Chest tube removal: end-inspiration or end-expiration? J Trauma 2001;50(4):674-677.

16. Fox SI. Fisiología humana. 12a ed. México: McGraw-Hill; 2011. p. 524-573.

17. Eren S, Esme H, Sehitogullari A, Durkan A. The risk factors and management of posttraumatic empyema in trauma patients. Injury 2008;39(1):44-49.

18. Karmy-Jones R, Holevar M, Sullivan RJ, Fleisig A, Jurkovich GJ. Residual hemothorax after chest tube placement correlates with increased risk of empyema following traumatic injury. Can Respir J 2008;15(5):255-258.

19. Hernández LA, Ruiz GJ, Escamilla AC. Epidemiología del trauma. En: Morales JL, editor. Tratado de cirugía general. 2a ed. México: El Manual Moderno; 2008. p. 1047-1054.

20. Belzunegui T. Protocolo de drenaje torácico del servicio de urgencias del Hospital de Navarra. Técnicas de Urgencias. Edición electrónica. España: Universidad de Navarra; 2000.

21. Rahman NM, Maskell NA, Davies CW, et al. The relationship between chest tube size and clinical outcome in pleural infection. Chest 2010;137(3):536-543. doi: 10.1378/chest.09-1044.

22. Cueto LGA. Colocación de un drenaje torácico. Neumosur 1994;6(2):40-42.

23. Yadav K, Jalili M, Zehtabchi S. Management of traumatic occult pneumothorax. Resuscitation 2010;81(9):10631068. doi: 10.1016/j.resuscitation.2010.04.030.

24. Cothren CC, Biffl WL, Moore EE. Traumatismos. En: Brunicardi FC, Andersen DK, Billiar TR, et al., editores. Principios de cirugía. 9a ed. México: McGraw-Hill; 2011. p. 135-195.

\section{$\triangle$ Correspondencia:}

Dr. Alberto Pérez Cantú Sacal, Hospital Central de la Cruz Roja Mexicana. Ejército Nacional Núm. 1032, Colonia Los Morales Polanco. México, D.F., 11500. Teléfono: 53951111 extensión 214

Correo electrónico: dr.apcantu@gmail.com

Los autores declaran no tener conflicto de intereses. 